\title{
A model to predict the propagation of Ebola
}

\author{
Tong Zheng Jiang \\ North China Electric Power University \\ 689, Huadian Road, Baoding, Hebei Province 071003 \\ P.R.China \\ jiangtongzheng_001@163.com
}

\section{Keywords: Ebola; SIR model; Runge-Kutta method; comprehensive evaluation coefficient}

\begin{abstract}
By predicting the propagation of Ebola, we can find a better way to control the spread of the virus. Unlike traditional SIR model, we consider the patients under incubation period and antibody carriers after cure in our model. To begin with, we apply five classifications to sample individuals (i.e. the susceptible, borderline cases, confirmed cases, death cases and the cured cases), by which we establish a first order differential system to represent the disease propagation. By applying Runge-Kutta method to established system, we obtain a fitted curve. Then, to get the access to the detailed propagation parameters, the curve obtained are further fitted with overall profile of epidemic situation as well as top three mostly-infected countries, through which the future propagation is more predictable. Finally, we introduce the comprehensive evaluation coefficient of effective cover efficiency $\xi$. The result shows that when $\xi$ is $100 \%$, the time of disease controlled is 13 weeks. And when $\xi$ is 0 , the time of complete eradication of the disease is more than 50 weeks without any medical treatment. Thus, it is better to take a medical treatment of higher intensity.
\end{abstract}

\section{Introduction}

The severe Ebola virus disease (EVD), also known as hemorrhagic fever, and its case fatality rate is around $50 \%$. Case fatality rates have varied from $25 \%$ to $90 \%$ in past outbreaks[1]. Ebola spreads through human-to-human transmission via direct contact with the blood, secretions, organs or other bodily fluids of infected people. The incubation period is 2 to 21 days[2]. And what should be taken into concern is that humans are not infectious until they develop symptoms[3].

From 1976, EVD first appeared till now, there has been more than ten times outbreak. But the outbreak in 2014 is known hitherto as the most destructive outbreak because of its long duration and death toll. By the 4th of Feb,2015, it has confirmed 22495 cases of infection, deaths up to 8987 cases and Guinea(2975 confirmed,1944 dead),Liberia(8745 confirmed,3746 dead),Sierra Leone(10740 confirmed,3276 dead), these three countries epidemic is particularly prominent.

In order to predict the propagation of EVD, we first carry on the research of the Ebola virus infections model [4], looking at the previous results, Chowell and Nishiura(2015)[5] carried out a model about transmission dynamics and control of EVD, and a comparative review of Ebola in the context of past outbreaks and the ongoing epidemic in West Africa. But they did not address the influence of the suspected. Wang [6] and Yarus [7] both used the epidemic model to explore the spread of Ebola and SARS virus. But Wang only apply them to the SIR model without considering the reinfected people after recovery and he didn't give a clear and accurate definition of the people who are in the incubation period. And Yarus didn't consider the production of antibodies of human body after recovery and he failed to actually compare and analysis the model.

The difference between our model and theirs is that, we based on the core assumptions which are the fixed number population, a number of people could obtain antibody and divided people into groups of susceptible population, already infected population, deceased population, healed population which can be divided into people with antibodies and people without, and some infected people who are still in the incubation period which are asymptomatic and non-infectious[8]. According to the number of 
population we establish the first order equations and solve them by Runge-Kutta method[9]. We will begin to integrate WHO weekly epidemic data from March, 22nd, acquiring the accumulated diagnosed numbers and deaths[10]. Then combine and analysis the overview of the epidemic and the data from the most infected countries based on the model so as to get a rough transmissions trend.

Based on the model above, we assume bringing the medical control intervebtions in the 44th week and also bringing in evaluation parameters, considering the influence of drugs and vaccine on various people so as to get the ideal trend curve of the Ebola transmission after medical control.

\section{Assumption and Explanations}

1. The population involved stays constant. (no births or unrelated deaths)

2. The susceptible and the infected almost go about their daily lives so the infected people are randomly distributed over the area.

3. As a matter of fact, some of individuals that survive from Ebola could get immunity.

4. Humans in the incubation period are not infectious until they develop symptoms.

5. Assuming the patients' medication cycle is one week.

\section{Definitions:}

Table 1 Model parameters

\begin{tabular}{cc}
\hline Symbol & Meaning \\
\hline$\lambda$ & Effective contact rate in one week \\
$\mu$ & Mortality rate in one week \\
$\beta$ & Recovery rate in one week \\
$\delta$ & Rate of rehabilitate with antibodies \\
$\varepsilon$ & Production rate of Suspected cases in one week \\
$p_{1}$ & Diagnosis rate of suspected cases in one week \\
$p_{2}$ & Probability of new drugs are working \\
$N$ & Probability of vaccine make susceptible people have antibody \\
$\xi$ & The total population in Epidemic area \\
\hline
\end{tabular}

\section{The infectious disease model based on pharmacokinetics}

The final purpose is to decide when is the latest intervention for the spread of disease. Before this, we need to according to our collected data from March 25, 2014 to February 4, 2015, to establish model. March 25, 2014 for the first day of our model, the Ebola virus propagation model.

Inspired by classical infectious disease model, in view of the Ebola virus spread, the population is divided into five types:

The total number of the susceptible group is described by $S(t)$;

The total number of the infected group is described by $I(t)$;

The total number of the recovered group is described by $R(t)$. Among them, according to whether with antibodies can be divided into two categories;

The total number of the suspected group is described by $Y(t)$;

The total number of the deceased group is described by $D(t)$.

For the deceased in incubation period, due to its asymptomatic embodies, and without infectivity, we temporarily to be classified as $S(t)$. Thus, according to the actual change in the spread of all kinds of people, we roughly drug pharmacokinetics of classical compartment model to describe. As shown in Fig. 1. 


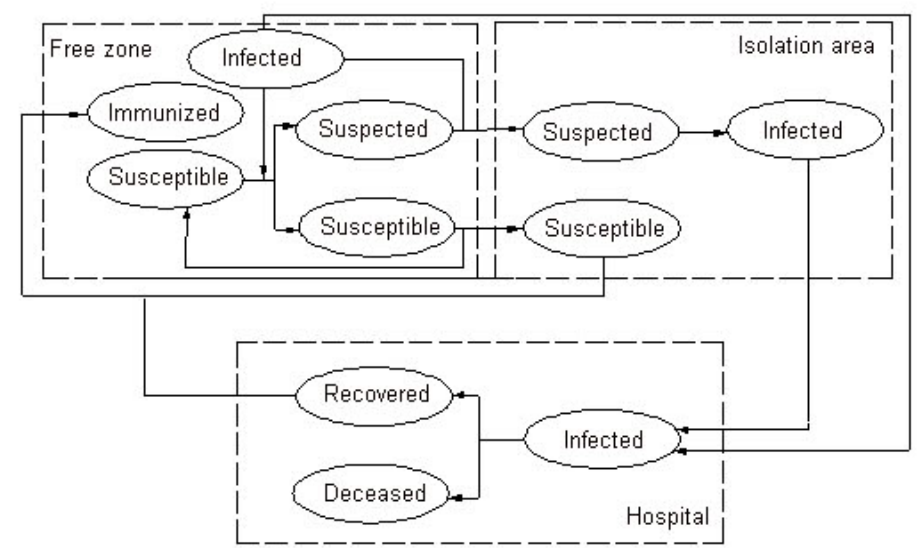

Figure 1. State transition diagram chamber model of the crowd.

Thus, we use a group of first order equations (Eq.1) to describe how the number of each group change so that we can calculate the spread of the virus in one week.

$$
\left\{\begin{array}{l}
\frac{d S(t)}{d t}=-\frac{\lambda}{N} \cdot S(t) \cdot I(t)-\delta \cdot S(t)+(1-\beta) \cdot R(t) \\
\frac{d I(t)}{d t}=\frac{\lambda}{N} \cdot S(t) \cdot I(t)-(\mu+\alpha) \cdot I(t) \\
\frac{d R(t)}{d t}=\mu \cdot I(t)-(1-\beta) \cdot R(t) \\
\frac{d D(t)}{d t}=\alpha \cdot I(t) \\
\frac{d Y(t)}{d t}=\delta \cdot S(t)-\varepsilon \cdot Y(t)
\end{array}\right.
$$

The above model reflected how Ebola influences the number of each group in in these group during its propagation. To get the trends of the virtus spread, first, we slove the first order equations by Runge-Kutta method and get the fitted curve of the accumulated infected and deceased numbers. Then combine it with the data from the most infected countries, Guinea, Liberia and Sierra Leone to get the parameters.

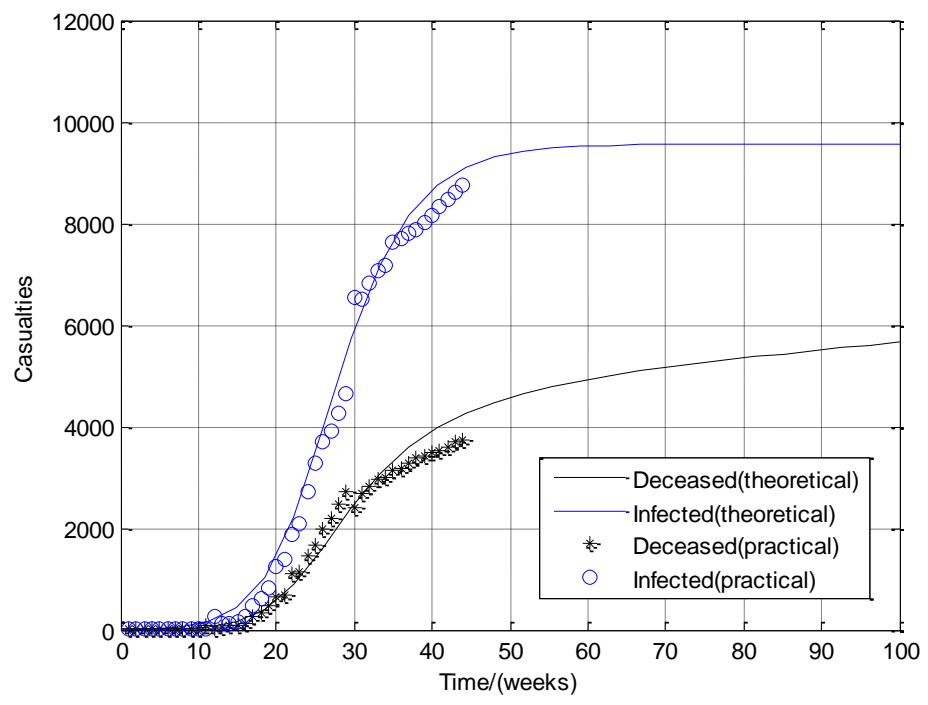

Figure 2. Trends chart of Liberia.

Using Liberia as an example, we can see, from the WHO first announced the disease, more than 40 
weeks up to now, the total number of the infected and the deceased curve, with the result of our model fitting degree is higher.

\section{The establishment of the medical intervention effectiveness evaluation model}

We assume that drugs and vaccines from the current (44 weeks) come into service. Introducing the comprehensive evaluation coefficient of effective cover efficiency $\xi$. We set up the success rate of the medicine to cure patients is $p_{1}$, the probability of antibody production after injection of the vaccine is $p_{2}$. To optimize the virus propagation model, the optimized had an impact on the susceptible, infected people, recovery crowd. We introduce the parameter $\xi$, set up model, to evaluate the influence of the system model. When we take medical interventions, respectively consider vaccines and drugs affect the infected and the recovered, thus we get a comprehensive treatment effectiveness evaluation model. And for different values of $\xi$, general analyze the spread of the epidemic and duration.

In order to measure the change of the number of people that has antibody, for some of the parameters are optimized model is as follows:

$$
\left\{\begin{array}{l}
\frac{d S(t)}{d t}=-\frac{\lambda}{N} \cdot S(t) \cdot I(t)-\delta \cdot S(t)+(1-\beta) \cdot R(t)-\xi \cdot p_{2} \cdot S(t) \\
\frac{d I(t)}{d t}=\frac{\lambda}{N} \cdot S(t) \cdot I(t)-(\mu+\alpha) \cdot I(t)-\xi \cdot p_{1} I(t) \\
\frac{d R(t)}{d t}=\mu \cdot I(t)-(1-\beta) \cdot R(t)+\xi \cdot p_{1} I(t) \\
\frac{d M(t)}{d t}=\beta \cdot R(t)+\xi \cdot p_{2} \cdot S(t)
\end{array}\right.
$$

Compared with the virus propagation model, when $\xi=1$, namely all patients to receive treatment. We are fitting out the optimized curve, as shown in fig. 3 . We assume $p_{1}=0.45, p_{2}=0.8$.

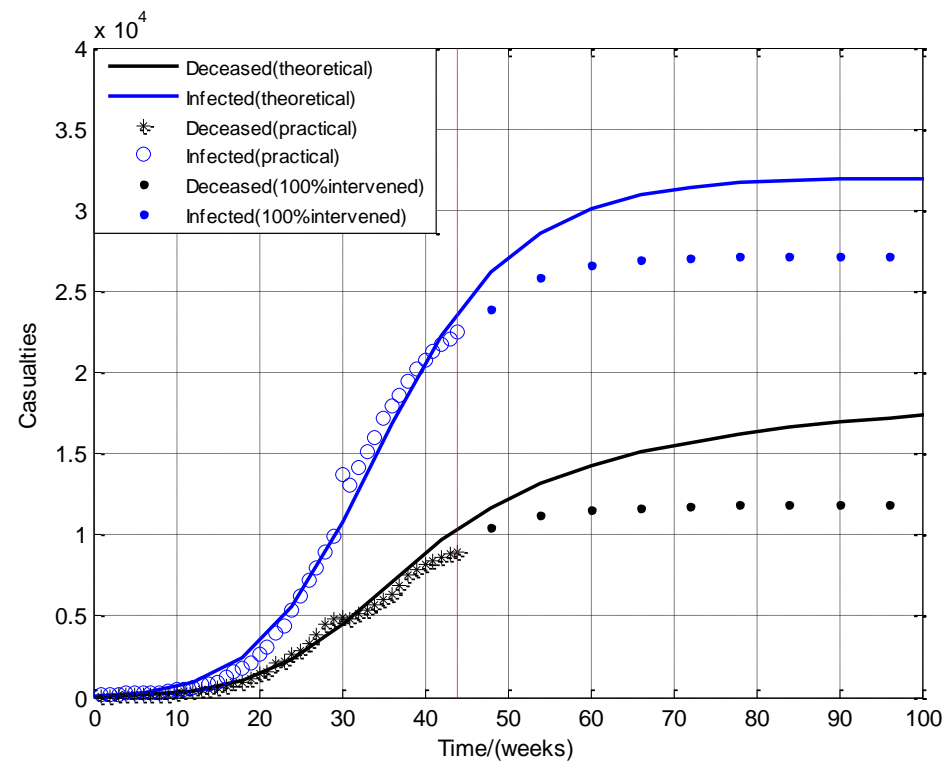

Figure 3. Trends chart of Total.

We can clearly see that after taking medical controls, in the ideal case, the rising trend of the total number of infections and deaths began to slow down. And after 13 weeks, it comes to be zero. According to the provisions of WHO, if there is no new case of infection appears within the period of 6 weeks which is twice as the Ebola longest incubation period(21 days), the epidemic can be declared as 
ended.

We carried out an evaluation coefficients analysis, for different coefficients, the time which the disease can be controlled in is also different. shown as the graph below :

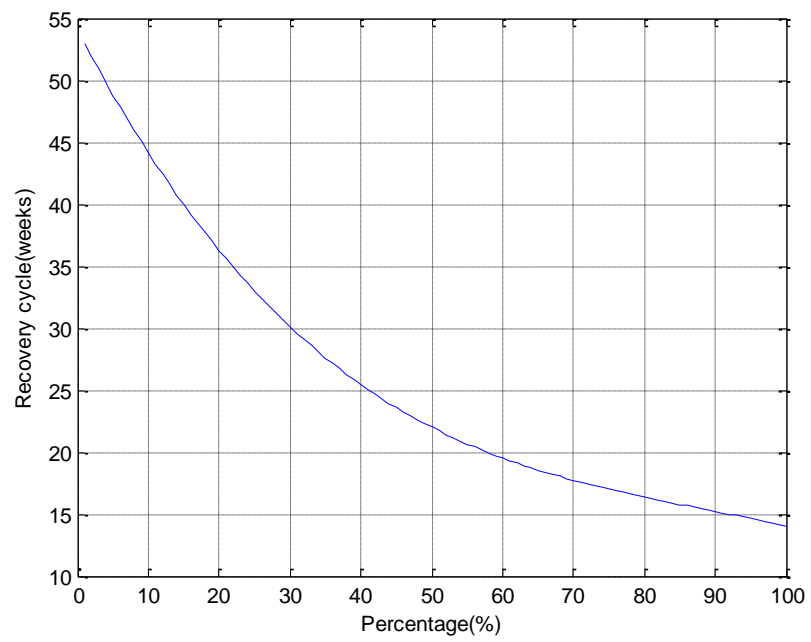

Figure 4. Recovery along with the changes of $\xi$

As can be seen from the graph, with the increasing assessment coefficients $\xi$, the period of patient's rehabilitation is reduced. When $\xi$ is $100 \%$, the time of disease controlled is 13 weeks. And when $\xi$ is 0 , the time of complete eradication of the disease is more than 50 weeks without any medical treatment. There is a notable difference between two sides. Thus, it is better to take a medical treatment of higher intensity.

After bringing in medical interventions, $p_{1}$ represents the recovery rate after taken medicine, we assume a constant number $p_{1}$, and the accumulated number of the deceased is 11849 . However, $p_{1}$ can not be constant in most situations. So when we change it to 0.4 , the deceased number comes to 11923 . Thus, the deceased number ranges with $p_{1}$.

\section{Reference}

[1] G. Katriel: Existence of Periodic Solutions for the Periodically Forced Sir Model. Vol 201, Issue 3, p. 335, 02 Aug 2014.

[2] Heinz Feldmann, Thomas W Geisbert. Ebola haemorrhagic fever. The Lancet . 2011 (9768)

[3] F. Chiappelli and A. Bakhordarian: Ebola translational science considerations. Journal of Translational Medicine. January 2015.

[4] Q.Y. Jiang, J.X. Xie and J. Ye: Mathematical model (Higher Education Press, China 2003), p. 135 (In Chinese)

[5] Gerardo Chowell and Hiroshi Nishiura: Transmission dynamics and control of Ebola virus disease (EVD): a review. BMC Medicine. Oct 2014

[6] Y.F. Wang, Y. Tian and Q. Wang: establishment and analysis of the SARS mathematical models. (Journal Of Engineering Mathematics, Dec 2003).

[7] Z. Yarus. A Mathematical look at the Ebola Virus. 11, 2012.

[8] Estimating the Future Number of Cases in the Ebola Epidemic-Liberia and Sierra Leone, 2014-2015. Morbidity and Mortality Weekly Report. Supplement / Vol. 63 /No. 3.September 26, 2014.

[9] S.K. Si and X.J. Sun: Mathematical modeling algorithm and application (National Defense Industry Press, China 2014), p.117 (In Chinese)

[10] Data of WHO. Feb 2015, Web site : http://www.who.int/mediacentre/factsheets/fs103/en/ 